\title{
Print still favored medium for biomedical information
}

The 91st annual meeting of the Medical Library Association, held May 31-June 6, 1991, in San Francisco, will long be remembered for its provocative and stimulating opening session. Two distinguished physicians, George D. Lundberg II, M.D. and Stephen P. Lock, M.D., editors of the prominent medical journals The Journal of the American Medical Association (JAMA) and the British Medical Journal, respectively, addressed a topic of great importance to health sciences librarianship-the future of biomedical information.

The dialogue begun by the speakers was further augmented by questions posed from a panel of prominent medical librarians, including Rachael Anderson, Ruth Holst, J. Michael Homan, Nina W. Matheson, and Bernie Todd Smith.

Both Lundberg and Lock believe that information, particularly in printed form, is now and will continue to be essential to the advancement of medicine. Lundberg predicted that at the end of this millennium, physicians, medical students, clinical scientists, and allied health workers will continue to receive most of their new medical knowledge from printed periodicals. Alternate forms of information, such as videotapes, CD-ROM, and formal education, will merely supplement rather than supplant traditional methods.

What then is the concern for the future of biomedical information? According to Lundberg, "ethical issues" will determine the real future of scientific information.

Lock agreed, stressing the importance of the integrity of editors and authors. He was more pessimistic about the future, however, believing that "as long as society continues to reward scientists on the basis of the 'quantity' of what they publish rather than 'quality,' the situation will continue to remain a mess."

Lundberg and Lock contend that editors and authors must share the ethical responsibility of biomedical information and publication. They maintain that editors of professional publications must be the "conscience of their professions." Critical areas of concern for both editors and authors include confidentiality, conflicts of interest, the peer review process, corrections and retractions, and instructions to authors.

Lock and Lundberg have initiated procedures to improve the integrity of scientific publication. Two strategies they both emphasize are "peer review" and a greater awareness of "conflict of interest." Lock believes that "structured abstracts," which were recently introduced in both the British Medical Journal and JAMA, will help alleviate some of the fraudulent problems occurring in scientific pub- lishing. None of these measures guarantee accuracy in scientific publication, but they are a start in the right direction, and they are the best they can do right now. Both editors agreed that "blind reviews" are the next step to pursue. They also maintain that it is the role of an editor to provide a forum for discussion. Editors must be concerned with publishing articles that are "in the public interest" rather than those that are primarily in "self interest." The medical journal also has an obligation to provide facts, a forum, and to take an editorial position on topics and then "campaign." This is where editors, according to Lock, can get into trouble: "They must base their campaigns on facts not on emotion." -Kathryn Hoffman, Director, University of Texas Southwestern Medical Center at Dallas Library

$E d$. note: Kathryn Hoffman served as the associate chair on the 1991 National Program Committee of the Medical Library Association.

\section{MLA tapes available}

All major sessions of MLA's meeting were taped and are available from: Audio Transcripts Ltd., 335 S. Patrick St., Suite 220, Alexandria, VA 22314; (703) 549-7334. The single cassette price is $\$ 12.00$.

Programs of interest to ACRL members are:

- Plenary Session I: "The Future of Biomedical Information." (Tape GS2, two cassettes.)

- Plenary Session III: "Scientists" Needs and Opportunities for Communication." (Tape GS4.)

- The Unified Medical Language System (UMLS). Knowledge Sources: Applications to Library and Information Science. (Tape 26, two cassettes.)

- Introduction to Publishing for Health Sciences Librarians: An Expert Panel Presentation. (Tape 27, two cassettes.)

- SatelLife: Building Electronic Bridges to Medical Libraries in the Developing World. (Tape 29, two cassettes.)

- Librarians, Legislation, Lobbying: We Can Make a Difference. (Tape 30, two cassettes.)

- Fraudin Scientific Publishing. (Tape 15.)

- Student Computer Laboratories and Networking. (Tape 04.)

- Wheat from the Chaff: Librarians as Information Evaluators. (Tape 10.) 


\section{We'll give you a spectacular view of the science world.}

\section{The New v.3.0 Search and Retrleval Software - Avallable October 1991}

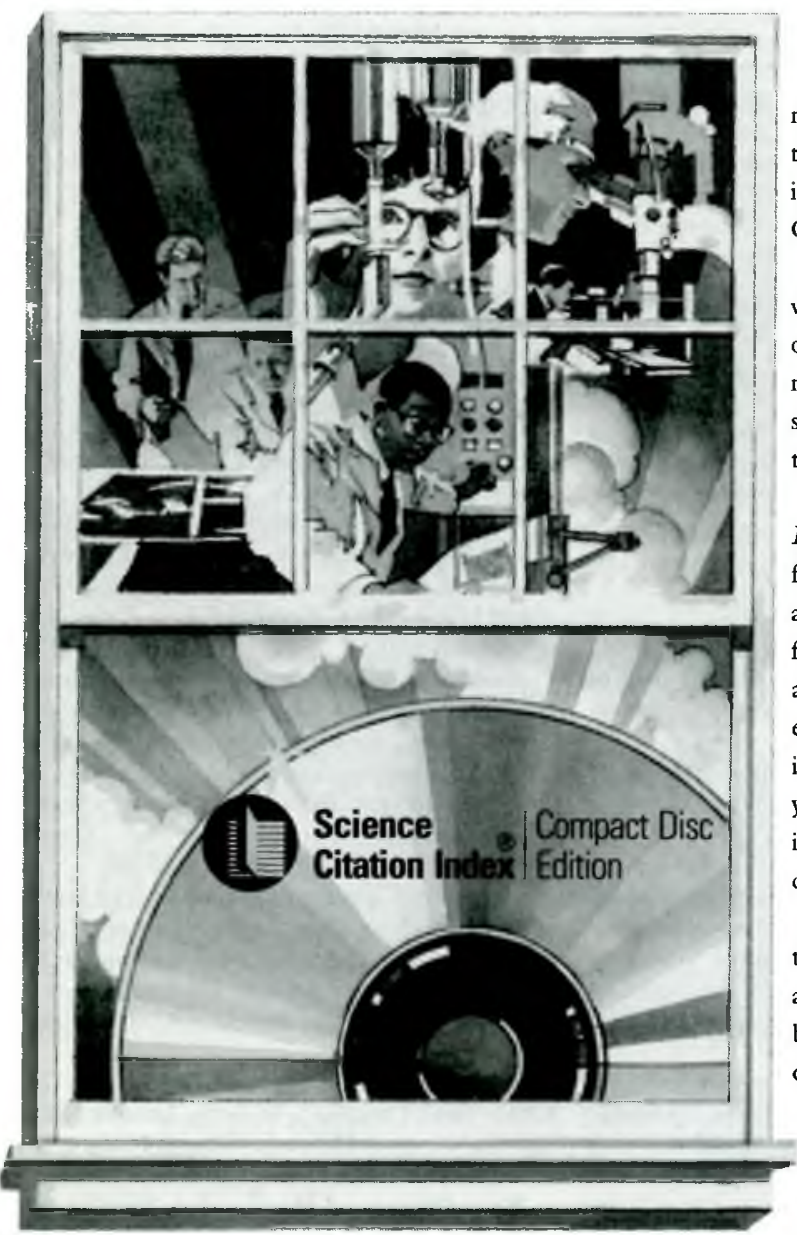

The best thing is, you need go no farther than your computer terminal-where you'll see it all in the Science Citation Index Compact Disc Edition.

With simple keystrokes, you're viewing the bibliographic records of 3,100 leading science journals... and running lightning-fast searches to retrieve data on thousands of research topics.

Another keystroke activates Related Records, the powerful searching mechanism available only from ISI. When you find one pertinent article, you're automatically led to many more, even if they have no title words in common! It's information you'd find no other wayinformation that could have a dramatic impact on your research.

Want to see how spectacular the vicw is from here? Reserve a free trial copy of the $\mathrm{SCI}^{\oplus} \mathrm{CDE}$ by calling $800-336-4474$, operator $\mathbf{R 4 1 9}$, or write the ISI office nearest you.

Institute for Scientific Information

3501 Market Strest, Philadelphia, PA 19104 U.S.A. Tolephone: (215) 386-0100, Fax: (215)386-6362 ISI European Branch: 132 High Street, Uxbridge, UB8 1DP U. K. Telephone: +44-895-270016 Fax: +44-895-256710 Tөlex: 933693 UKISI

The ISI Indexes...Gateway to the Literature of Science

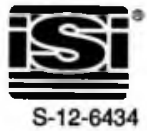

